\title{
On the Distribution of the Zeros of Generalized Airy Functions
}

\author{
By V. B. Headley and V. K. Barwell
}

\begin{abstract}
We give tables of zeros and values of the generalized Airy functions introduced by Swanson and Headley [SIAM J. Appl. Math., v. 15, 1967, pp. 1400-1412]. The tables enable us to sharpen substantially results on the distribution of the zeros. We show that the nonreal zeros are asymptotically close to the boundary rays of the sectors obtained in the paper cited. We conjecture from the numerical evidence that the zeros monotonically approach the rays.
\end{abstract}

1. Introduction. In a previous article [9], Swanson and one of the present authors discussed a pair of linearly independent solutions of the ordinary differential equation

$$
d^{2} u / d z^{2}-z^{n} u=0
$$

where $z$ is a complex variable and $n$ is a positive integer. This differential equation is of interest because, inter alia, it is the simplest ordinary differential equation of the second order with a turning point of order $n$. It is thus a natural comparison equation to use when seeking uniform asymptotic expansions in unbounded regions containing a turning point of order $n$ for general second-order equations. The equation we are discussing also arises in various physical situations; for example, the propagation of waves in varying media [1].

In [9] two linearly independent solutions $A_{n}(z), B_{n}(z)$ were chosen in such a manner that they became, for $n=1$, the well-known Airy functions discussed in detail by Miller [3], Olver [6], Swanson [8], and others. It was shown [9] that the zeros of $A_{n}(z), B_{n}(z)$ and their derivatives were restricted to certain sectors of the complex $z$ plane. Since it is known [6] that the real zeros of $A_{1}(z)$ and $B_{1}(z)$ lie along the negative real axis, we asked ourselves whether we could obtain more precise information regarding the asymptotic distribution of the large nonreal zeros of $A_{n}(z), B_{n}(z)$ and their derivatives. This information, if obtainable, would permit much sharper estimates of the maximum domain of validity for the uniform asymptotic expansions mentioned above. We, therefore, wrote a computer program to calculate the zeros, using reversion of the asymptotic series for the solutions of (1). (The tables, which also include the zeros of the derivatives $A_{n}^{\prime}(z), B_{n}^{\prime}(z)$ and the values of $A_{n}(z), B_{n}(z)$ at the zeros of $A_{n}^{\prime}(z), B_{n}^{\prime}(z)$, appear at the end of this paper.) By inspecting these results, we were led to conjecture and prove that the large zeros $z_{m}$ lie close to the farther boundary ray of each of the sectors discovered in [9]; indeed there is empirical evidence that in each of these sectors, $\operatorname{larg}\left(z_{m}\right) \mid$ increases strictly with $m$. Inspection of the tables also reveals

Received June 11, 1974; revised September 27, 1974.

AMS(MOS) subject classifications (1970). Primary 65D20; Secondary 34C10.

Key words and phrases. Generalized Airy functions, tables of zeros, asymptotic distribution of zeros, turning points. 
that even the small zeros lie close to the appropriate boundary ray. For example, if we consider $A_{n}(z)$, we see that $\arg \left(z_{1}\right)$ is within 0.04 radians of the boundary so that more than eighty percent of the sector is in fact zero-free. The results obtained in the present paper therefore constitute a substantial sharpening of known results, even in the classical case $n=1$.

To improve the accuracy of the small zeros, we used Aitken's method with ascending series, then the results were checked by comparing them with known results [3] for $n=1$ and by substituting into the ascending series and the asymptotic series for the solutions of (1).

2. Definitions and Background Material. As in [9], we define the functions $A_{n}$ and $B_{n}$ by the relations

$$
\begin{aligned}
& A_{n}(z)=p z^{1 / 2}\left[I_{-p}(\zeta)-I_{p}(\zeta)\right] \\
& B_{n}(z)=(p z)^{1 / 2}\left[I_{-p}(\zeta)+I_{p}(\zeta)\right]
\end{aligned}
$$

where

$$
\zeta=2 p z^{1 /(2 p)}, \quad p=1 /(n+2),
$$

and $I_{p}$ denotes the modified Bessel function of the first kind of order $p$. In the case $n=1$, the functions $A_{n}$ and $B_{n}$ reduce to the usual Airy functions $A i$ and $B i$, respectively.

It follows from the standard theory of Bessel functions [11] that $A_{n}(z)$ and $B_{n}(z)$ are real on the real axis, analytic in the finite complex plane, and are linearly independent solutions of the differential equation (1).

According to [9], the functions $A_{n}$ and $A_{n}^{\prime}$ have no real zeros for even $n$; but for odd $n$, they have an infinite sequence of simple zeros on the negative real axis with no finite accumulation point. Moreover, nonreal zeros of $A_{n}$ and $A_{n}^{\prime}$ are all contained in the region $\bigcup_{k} S_{k}$, where $k$ takes all integer values in the range $0<2 k<n+1$, and $S_{k}$ is the double sector

$$
\{z: 2 k \pi /(n+1)<|\arg z|<(2 k+1) \pi /(n+2)\} .
$$

An analogous result holds for $B_{n}$ and $B_{n}^{\prime}$; but in this case, the nonreal zeros lie in the region $\bigcup_{k} S_{k}^{\prime}$, where $k$ takes all integer values in the range $0<2 k<n+3$, and $S_{k}^{\prime}$ is the double sector

$$
\{z:(2 k-1) \pi /(n+2)<|\arg z|<(2 k-1) \pi /(n+1)\} .
$$

\section{Asymptotic Distribution of the Zeros.}

THEOREM 1. For each positive integer $n$, the zeros of $A_{n}$ or $A_{n}^{\prime}$ which lie in the double sector $S_{k} \quad(0<2 k<n+1)$ approach one of the rays $|\arg z|=$ $(2 k+1) \pi /(n+2)$. That is, if the zeros in the sector $S_{k}$ are arranged in ascending order of absolute value: $\left|z_{k 1}\right|<\left|z_{k 2}\right|<\ldots<\left|z_{k m}\right|<\ldots$, then

$$
\lim _{m \rightarrow \infty}\left|\arg z_{k m}\right|=\frac{(2 k+1) \pi}{n+2} \quad\left(k=1,2, \ldots,\left[\frac{n+1}{2}\right]\right) .
$$


Moreover, the distance between successive zeros approaches zero as $m$ approaches infinity.

Proof. For a zero $z$ of $A_{n}$ or $A_{n}^{\prime}$ in the upper half-plane, let $\zeta=Z e^{(k+1 / 2) \pi i}$. Then [11, pp. 77-78]

$$
I_{p}(\zeta)=e^{(k+1) p \pi i} I_{p}\left(Z e^{-i \pi / 2}\right)=e^{(k+1 / 2) p \pi i} J_{p}(Z) \quad(-\pi / 2<\arg Z \leqslant \pi),
$$

and

$$
I_{-p}(\zeta)=e^{-(k+1 / 2) p \pi i} J_{-p}(Z) \quad(-\pi / 2<\arg Z \leqslant \pi) .
$$

Making use of the relation [11, p. 74]

$$
J_{-p}(Z)=J_{p}(Z) \cos p \pi-Y_{p}(Z) \sin p \pi
$$

where $Y_{p}$ is Weber's Bessel function of the second kind of order $p$, we see that, for $-\pi / 2<\arg Z \leqslant \pi$,

$$
\begin{aligned}
I_{-p}(\zeta)-I_{p}(\zeta)= & {\left[e^{-(k+1 / 2) p \pi i} \cos p \pi-e^{(k+1 / 2) p \pi i}\right] J_{p}(Z) } \\
& -e^{-(k+1 / 2) p \pi i} Y_{p}(Z) \sin p \pi
\end{aligned}
$$

It follows that there exists a complex number $\alpha$ such that

$$
I_{-p}(\zeta)-I_{p}(\zeta)=J_{p}(Z) \cos \alpha-Y_{p}(Z) \sin \alpha .
$$

To see this, we note that the equation $\tan \alpha=\omega$ has solutions given by

$$
e^{i \alpha}= \pm \sqrt{(1+i \omega) /(1-i \omega)}
$$

hence $\alpha$ is well defined when $\omega \neq \pm i$. If we now set

$$
\tan \alpha=\omega=\frac{e^{-(k+1 / 2) p \pi i} \sin p \pi}{e^{-(k+1 / 2) p \pi i} \cos p \pi-e^{(k+1 / 2) p \pi i}}=\frac{\sin p \pi}{\cos p \pi-e^{(2 k+1) p \pi i}}
$$

we see that the exceptional values $\pm i$ of $\omega$ occur when

$$
\cos p \pi=\cos (2 k+1) p \pi \text { and } \sin p \pi= \pm \sin (2 k+1) p \pi
$$

that is, when $(2 k+1) p \pi=2 l \pi \pm p \pi(l=1,2,3, \ldots)$. But $p=2 /(n+2)$, hence the exceptional values of $\omega$ only occur when $2 k+1=l(n+2)(l=1,2,3, \ldots)$. Since $0<2 k<n+1$, it follows that the exceptional values of $\omega$ cannot occur. Thus, a complex number $\alpha$ can always be found satisfying (7). It is known [11, pp. 505506] that the large zeros $Z_{k m}$ of $J_{p}(Z) \cos \alpha-Y_{p}(Z) \sin \alpha$ have the asymptotic expansion

$$
Z_{k m} \sim b_{k m}-\frac{4 p^{2}-1}{8 b_{k m}}-\frac{\left(4 p^{2}-1\right)\left(28 p^{2}-31\right)}{384 b_{k m}^{3}}-\ldots, \quad(m \rightarrow \infty),
$$

where $b_{k m}=(m+1 / 2 p-1 / 4) \pi-\alpha$. It is clear from (9) that $\alpha$ is independent of $m$. Let $\alpha=\alpha_{1}+i \alpha_{2}$. Then it follows from (10) that $\operatorname{Im}\left(Z_{k m}\right)=-\alpha_{2}+O\left(b_{k m}^{-1}\right)$ $(m \rightarrow+\infty)$, 


$$
\operatorname{Re}\left(Z_{k m}\right)=(m+1 / 2 p-1 / 4) \pi-\alpha_{1}+O\left(b_{k m}^{-1}\right) \quad(m \rightarrow+\infty)
$$

hence $\lim _{m \rightarrow \infty} \operatorname{Im}\left(Z_{k m}\right) / \operatorname{Re}\left(Z_{k m}\right)=0$. The corresponding zero $z_{k m}$ of $A_{n}(z)$ satisfies the relation

$$
\arg z_{k m}=\frac{n+2}{2} \arg Z_{k m}+\frac{(2 k+1) \pi}{n+2}
$$

It follows that $\lim _{m \rightarrow \infty} \arg z_{k m}=(2 k+1) /(n+2)$. With $k$ fixed, abbreviate $Z_{k m}$, $z_{k m}, b_{k m}$ by $Z_{m}, z_{m}, b_{m}$, respectively. Let $Z_{m+1}-Z_{m}=\epsilon_{m}$. Then $\epsilon_{m}=\pi+$ $O\left(b_{m}^{-1}\right)(m \rightarrow \infty)$.

By (4) and the definition of $Z$ we must have

$$
z_{m}=\left[Z_{m} /(2 p)\right]^{2 p} e^{(2 k+1) p \pi i}
$$

and

$$
z_{m+1}=\left[Z_{m+1} /(2 p)\right]^{2 p} e^{(2 k+1) p \pi i}
$$

Let

$$
t_{m}=\left(\frac{Z_{m}+\epsilon_{m}}{2 p}\right)^{2 p}-\left(\frac{Z_{m}}{2 p}\right)^{2 p} \quad(m=1,2,3, \ldots) .
$$

Then

$$
\begin{aligned}
t_{m} & =\left(\frac{Z_{m}}{2 p}\right)^{2 p}\left[\left(1+\frac{\epsilon_{m}}{Z_{m}}\right)^{2 p}-1\right] \\
& =\left(\frac{Z_{m}}{2 p}\right)^{2 p}\left[\frac{2 p \epsilon_{m}}{Z_{m}}+\frac{2 p(2 p-1) \epsilon_{m}^{2}}{2 Z_{m}^{2}}+O\left(\frac{\epsilon_{m}}{Z_{m}}\right)^{3}\right] \\
& =\left(\frac{2 p}{Z_{m}}\right)^{1-2 p} \epsilon_{m}+O\left(\frac{1}{Z_{m}}\right)^{2-2 p} \quad(m \rightarrow \infty) .
\end{aligned}
$$

It follows that $\lim _{m \rightarrow \infty} t_{m}=0$; and, therefore, $\lim _{m \rightarrow \infty}\left(z_{m+1}-z_{m}\right)=0$. Since the zeros are symmetrically placed with respect to the real axis, the proof of the theorem is now complete.

THEOREM 2. For each positive integer $n$, the zeros of $B_{n}$ or $B_{n}^{\prime}$ which lie in the double sector in $S_{k}^{\prime}(0<2 k<n+3)$, approach one of the rays $|\arg z|=$ $(2 k-1) \pi /(n+2)$. Moreover, if $z_{m}$ and $z_{m+1}$ are successive zeros in $S_{k}^{\prime}$, with $\left|z_{m}\right|<$ $\left|z_{m+1}\right|(m=1,2,3, \ldots)$, then

$$
\lim _{m \rightarrow \infty}\left|z_{m+1}-z_{m}\right|=0 .
$$

The proof will be omitted since it is similar to that of Theorem 1 .

REMARK. We conjecture from the numerical and asymptotic evidence that if $z_{m}$ is a zero of $A_{n}$ or $A_{n}^{\prime}$ in the sector $S_{k}(0<2 k<n+1)$, then $\left|\arg z_{m}\right|$ increases strictly with $m$; and that if $z_{m}$ is a zero of $B_{n}$ or $B_{n}^{\prime}$ in the sector $S_{k}^{\prime}(0<2 k<n+3)$, then $\left|\arg z_{m}\right|$ decreases strictly with $m$. 
4. Computation of the Zeros. We shall find approximations to the zeros of $A_{n}(z)$ by reverting the asymptotic series for the general cylinder function given by (7). Accordingly, let $\zeta_{1}$ be a zero of the cylinder function

$$
J_{p}\left(\zeta_{1}\right) \cos \alpha-Y_{p}\left(\zeta_{1}\right) \cos \alpha
$$

where

$$
\begin{gathered}
p=1 /(n+2), \quad \zeta_{1}=2 p z^{1 /(2 p)} e^{-(k+1 / 2) \pi i}, \\
\tan \alpha=\frac{\sin p \pi}{\cos p \pi-\exp [(2 k+1) p \pi i]},
\end{gathered}
$$

and $k$ is an integer in the range $0<2 k<n+1$. It follows from known results [11, pp. 504-506] that the $r$ th zero of (11) is given by

$$
\psi=\zeta_{1}-1 / 2 p \pi+1 / 4 \pi+\alpha-r \pi
$$

where (14) is obtained by integrating the asymptotic series

$$
1-\frac{d \psi}{d \zeta_{1}} \sim\left[\sum_{m=0}^{\infty}\{1 \cdot 3 \cdot 5 \ldots(2 m-1)\} \frac{(p, m)}{2^{m} \zeta_{1}^{2 m}}\right]^{-1}
$$

with

$$
(p, 0)=1, \quad(p, m+1)=\left[\frac{4 p^{2}-(2 m+1)^{2}}{2^{2}(m+1)}\right](p, m) \quad(m=0,1,2, \ldots) .
$$

It should be noted that if $z$ is a zero of $A_{n}(z)$ in the double sector $\{z: 2 k \pi /(n+1)<$ $|\arg z|<(2 k+1) \pi /(n+2)\}$, then it follows from (12) that $\left|\arg \zeta_{1}\right|<\pi$; and, therefore, the asymptotic expansion (15) is applicable [11, p. 449]. On integrating (15) and making use of (14), we obtain the asymptotic representation

$$
\zeta_{1}-(k+1 / 2 p-1 / 4) \pi+\alpha \sim \sum_{m=1}^{\infty} \frac{b_{m+1}}{(2 m-1) \zeta_{1}^{2 m-1}}
$$

where

$$
\begin{aligned}
& b_{1}=1, \quad b_{m}=-\sum_{j=2}^{m} a_{j} b_{m+1-j} \quad(m=2,3,4, \ldots), \\
& a_{1}=1, \quad a_{m+1}=\frac{(2 m-1)\left[4 p^{2}-(2 m-1)^{2}\right]}{2^{3} m} a_{m} \quad(m=0,1,2, \ldots) .
\end{aligned}
$$

Finally, we set $\beta=(k+1 / 2 p-1 / 4) \pi-\alpha$ and expand $z_{1}$ as a descending series in $\beta$. It turns out that if we go as far as the term in $\beta^{-9}$, we obtain results accurate to eight decimal places for $r \geqslant 4$.

To improve the accuracy of the first three zeros in each sector, we start with the ascending series obtained in [9], writing it in the form 


$$
t=p \Gamma(p) \sum_{m=0}^{\infty} \frac{t^{m(n+2)}}{m !(m+1-p)}-\sum_{m=1}^{\infty} \frac{t^{m(n+2)+1}}{m ! \Gamma(m+1+p)},
$$

where $t=p^{2 p} z$ and $z$ is a zero of $A_{n}(z)$ in the upper half of the double sector $S_{k}$. We now perform the rotation

$$
t_{1}=t / w, \quad w=\exp [(2 k+1) p \pi]
$$

so as to bring the zero as close as possible to the positive real axis. Finally, we use

(17) in the form

$$
t_{1} \approx \frac{1}{w} p \Gamma(p) \sum_{m=0}^{L} \frac{t^{m(n+2)}}{m ! \Gamma(m+1-p)}-\sum_{m=1}^{L} \frac{t^{m(n+2)+1}}{m ! \Gamma(m+1+p)}
$$

to generate successive approximations to $t_{1}$ for $L=0,1,2, \ldots$, each iterate of $t_{1}$ being sharpened by Aitken's $\Delta^{2}$-method before substitution into (19) for the next approximation.

The zeros of $A_{n}^{\prime}(z), B_{n}(z), B_{n}^{\prime}(z)$ were computed similarly by making suitable changes in (11)-(19).

The tables at the end of the paper were prepared from computer printout, hence a slight change in notation has been made. Instead of $A_{N}(Z), B_{N}(Z), A_{N}^{\prime}(Z), B_{N}^{\prime}(Z)$, the tables have $A N(Z), B N(Z), A N^{\prime}(Z), B N^{\prime}(Z)$. The zeros of $A N(Z)$ and $A N^{\prime}(Z)$ lie in the double sectors

$$
\{Z: 2 I \pi /(N+1)<|\arg (Z)|<(2 I+1) \pi /(N+2)\},
$$

where $I$ takes all integer values in the range $0<2 I<N+1$. The zeros of $B N(Z)$ and $B N^{\prime}(Z)$ lie in the double sectors

$$
\{Z:(2 I-1) \pi /(N+2)<|\arg (Z)|<(2 I-1) \pi /(N+1)\},
$$

where $I$ takes all integer values in the range $0<2 I<N+3$. In each sector, the zeros are listed in ascending order of their absolute value. We give only the first ten zeros in each sector, but we note that the technique used gives accurate results for all the zeros.

Department of Mathematics

Brock University

St. Catharines, Ontario, Canada

Department of Applied Analysis and Computer Science

University of Waterloo

Waterloo, Ontario, Canada

1. H. BREMMER, Terrestrial Radio Waves. Theory of Propagation, Elsevier, New York, 1949. MR 11, 295.

2. DONALD LUDWIG, "Uniform asymptotic expansions for wave propagation and diffraction problems," SIAM Rev., v. 12, 1970, pp. 325-331. MR 42 \#1407.

3. J. C. P. MILLER, The Airy Integral, Giving Tables of Solutions of the Differential Equation $y^{\prime}=x y$, British Association for the Advancement of Science, Mathematical Tables, Part-volume B., Cambridge Univ. Press, Cambridge, 1946. MR 8, 353.

4. F. W. J. OLVER, "A new method for the evaluation of zeros of Bessel functions and of other solutions of second-order differential equations," Proc. Cambridge Philos. Soc., v. 46, 1950, pp. 570-580. MR 12, 288.

5. F. W. J. OLVER, "A further method for the evaluation of zeros of Bessel functions and some new asymptotic expansions for zeros of functions of large order," Proc. Cambridge Philos. Soc., v. 47, 1951, pp. 699-712. MR 13, 283. 
6. F. W. J. OLVER, "The asymptotic expansion of Bessel functions of large order," Philos. Trans. Roy. Soc. London Ser. A, v. 247, 1954, pp. 328-368. MR 16, 696.

7. F. W. J. OLVER, Asymptotics and Special Functions, Academic Press, New York and London, 1974.

8. C. A. SWANSON, Properties of Airy Functions, Technical Report, California Institute of Technology, Pasadena, Calif., 1956.

9. C. A. SWANSON \& V. B. HEADLEY, “An extension of Airy's equation," SIAM J. Appl. Math., v. 15, 1967, pp. 1400-1412. MR 37 \#82.

10. WOLFGANG WASOW, Asymptotic Expansions for Ordinary Differential Equations, Wiley, New York a: d London, 1965. MR 34 \#3041.

11. G. N. WATSON, A Treatise on the Theory of Bessel Functions, 2nd ed., Cambridge Univ. Press, Lambridge, 1952. 
TABLE 1

ZEROS OF AN(Z) AN $^{\prime}(Z)$ AT THESE ZEROS

\begin{tabular}{|c|c|c|c|c|c|c|}
\hline $\mathbf{N}$ & I & $\mathbf{K}$ & ABSOLUTE VALUE & ARGUMENT & ABSOLUTE VALUE & ARGUMENT \\
\hline \multirow[t]{10}{*}{1} & 1 & 1 & 2.33810741 & 3.14159265 & 0.70121082 & 0.0 \\
\hline & & 2 & 4.08794944 & 3.14159265 & 0.80311137 & 3.14159265 \\
\hline & & 3 & 5.52055983 & 3.14159265 & 0.86520403 & 0.0 \\
\hline & & 4 & 6.78670809 & 3.14159265 & 0.91085074 & 3.14159265 \\
\hline & & 5 & 7.94413359 & 3.14159265 & 0.94733571 & 0.0 \\
\hline & & 6 & 9.02265086 & 3.14159265 & 0.97792281 & 3.14159265 \\
\hline & & 7 & 10.04017434 & 3.14159265 & 1.00437012 & 0.0 \\
\hline & & 8 & 11.00852430 & 3.14159265 & 1.02773869 & 3.14159265 \\
\hline & & 9 & 11.93601556 & 3.14159265 & 1.04872065 & 0.0 \\
\hline & & 10 & 12.82877675 & 3.14159265 & 1.06779386 & 3.14159265 \\
\hline \multirow[t]{10}{*}{2} & 1 & 1 & 2.19034316 & 2.32053093 & 0.70687774 & 0.37569934 \\
\hline & & 2 & 3.32181119 & 2.34053384 & 0.86594896 & -2.75663573 \\
\hline & & 3 & 4.15977716 & 2.34619170 & 0.96820857 & 0.38772177 \\
\hline & & 4 & 4.85595843 & 2.34885034 & 1.04580284 & -2.75255596 \\
\hline & & 5 & 5.46440016 & 2.35039341 & 1.10925011 & 0.38980335 \\
\hline & & 6 & 6.01168328 & 2.35140096 & 1.16339621 & -2.75128761 \\
\hline & & 7 & 6.51319779 & 2.35211043 & 1.21090419 & 0.39065874 \\
\hline & & 8 & 6.97879729 & 2.35263703 & 1.25304069 & -2.75067119 \\
\hline & & 9 & 7.41523962 & 2.35304338 & 1.29198370 & 0.39112430 \\
\hline & & 10 & 7.82739765 & 2.35336642 & 1.32738810 & -2.75030704 \\
\hline \multirow[t]{10}{*}{3} & 1 & 1 & 2.05058948 & 1.84553066 & 0.65135310 & 0.60002539 \\
\hline & & 2 & 2.85762476 & 1.86757748 & 0.83061108 & -2.52617108 \\
\hline & & 3 & 3.42040924 & 1.87384927 & 0.94959617 & 0.62002617 \\
\hline & & 4 & 3.87092999 & 1.87679966 & 1.04161221 & -2.51937602 \\
\hline & & 5 & 4.25419681 & 1.87851274 & 1.11788529 & 0.62349387 \\
\hline & & 6 & 4.59170790 & 1.87963151 & 1.18367563 & -2.51726294 \\
\hline & & 7 & 4.89563611 & 1.88041940 & 1.24190933 & 0.62491901 \\
\hline & & 8 & 5.17363567 & 1.88100423 & 1.29439784 & -2.51623591 \\
\hline & & 9 & 5.43087666 & 1.88145552 & 1.34234796 & 0.62569469 \\
\hline & & 10 & 5.67103832 & 1.88181432 & 1.38660815 & -2.51562920 \\
\hline \multirow[t]{5}{*}{4} & 1 & 1 & 1.93480106 & 1.53339438 & 0.59108461 & 0.74952439 \\
\hline & & 2 & 2.54957321 & 1.55427477 & 0.77419136 & -2.37255136 \\
\hline & & 3 & 2.96133373 & 1.56023389 & 0.89832191 & 0.77488084 \\
\hline & & 4 & 3.28288202 & 1.56303894 & 0.99553466 & -2.36393369 \\
\hline & & 5 & 3.55155083 & 1.56466802 & 1.07684986 & 0.77927890 \\
\hline
\end{tabular}


TABle 1 (Continued)

ZEROS OF AN (Z)

\begin{tabular}{|c|c|c|c|c|c|c|}
\hline $\mathrm{N}$ & I & $\mathbf{K}$ & ABSOLUTE VALUE & ARGUMENT & ABSOLUTE VALUE & ARGUMENT \\
\hline \multirow[t]{5}{*}{4} & 1 & 6 & 3.78482274 & 1.56573204 & 1.14748965 & -2.36125365 \\
\hline & & 7 & 3.99244987 & 1.56648141 & 1.21038250 & 0.78108643 \\
\hline & & 8 & 4.18049055 & 1.56703768 & 1.26735289 & -2.35995103 \\
\hline & & 9 & 4.35299545 & 1.56746694 & 1.31962279 & 0.78207025 \\
\hline & & 10 & 4.51282190 & 1.56780823 & 1.36805515 & -2.35918153 \\
\hline \multirow[t]{10}{*}{4} & 2 & 1 & 1.93112085 & 2.60816427 & 0.83449806 & 0.25237644 \\
\hline & & 2 & 2.54860724 & 2.61366431 & 1.09446892 & -2.88407953 \\
\hline & & 3 & 2.96087343 & 2.61522715 & 1.27022404 & 0.25904449 \\
\hline & & 4 & 3.28260643 & 2.61596220 & 1.40778125 & -2.88182018 \\
\hline & & 5 & 3.55136466 & 2.61638896 & 1.52281620 & 0.26019683 \\
\hline & & 6 & 3.78468721 & 2.61666765 & 1.62273749 & -2.88111815 \\
\hline & & 7 & 3.99234607 & 2.61686392 & 1.71169494 & 0.26067026 \\
\hline & & 8 & 4.18040807 & 2.61700960 & 1.79227234 & -2.88077699 \\
\hline & & 9 & 4.35292805 & 2.61712202 & 1.86619960 & 0.26092791 \\
\hline & & 10 & 4.51276562 & 2.61721140 & 1.93469805 & -2.88057547 \\
\hline \multirow[t]{10}{*}{5} & 1 & 1 & 1.84076804 & 1.31208620 & 0.53687755 & 0.85639356 \\
\hline & & 2 & 2.33112483 & 1.33122107 & 0.71677225 & -2.26278230 \\
\hline & & 3 & 2.65017756 & 1.33669279 & 0.84055893 & 0.88551777 \\
\hline & & 4 & 2.89494587 & 1.33926942 & 0.93837590 & -2.25288391 \\
\hline & & 5 & 3.09684672 & 1.34076604 & 1.02072758 & 0.89056939 \\
\hline & & 6 & 3.27038435 & 1.34174361 & 1.09263147 & -2.24980564 \\
\hline & & 7 & 3.42356311 & 1.34243212 & 1.15691753 & 0.89264551 \\
\hline & & 8 & 3.56131261 & 1.34294322 & 1.21535689 & -2.24830946 \\
\hline & & 9 & 3.68690490 & 1.34333764 & 1.26914026 & 0.89377550 \\
\hline & & 10 & 3.80263359 & 1.34365122 & 1.31911122 & -2.24742562 \\
\hline \multirow[t]{10}{*}{5} & 2 & 1 & 1.83759911 & 2.23108481 & 0.80322026 & 0.43330355 \\
\hline & & 2 & 2.33032279 & 2.23830188 & 1.07398710 & -2.69984121 \\
\hline & & 3 & 2.64980327 & 2.24035617 & 1.25977208 & 0.44426948 \\
\hline & & 4 & 2.89472502 & 2.24132269 & 1.40648555 & -2.69612623 \\
\hline & & 5 & 3.09669918 & 2.24188391 & 1.52997263 & 0.44616415 \\
\hline & & 6 & 3.27027791 & 2.24225044 & 1.63778034 & -2.69497197 \\
\hline & & 7 & 3.42348220 & 2.24250857 & 1.73416001 & 0.44694253 \\
\hline & & 8 & 3.56124874 & 2.24270017 & 1.82177053 & -2.69441106 \\
\hline & & 9 & 3.68685302 & 2.24284803 & 1.90239877 & 0.44736615 \\
\hline & & 10 & 3.80259048 & 2.24296558 & 1.97731031 & -2.69407973 \\
\hline
\end{tabular}


TABLE 2

ZEROS OF AN'(Z)

AN(Z) AT THESE ZEROS

\begin{tabular}{|c|c|c|c|c|c|c|}
\hline $\mathbf{N}$ & I & $\mathrm{K}$ & ABSOLUTE VALUE & ARGUMENT & ABSOLUTE VALUE & ARGUMENT \\
\hline \multirow[t]{10}{*}{1} & \multirow[t]{10}{*}{1} & 1 & 1.01879297 & 3.14159265 & 0.53565666 & 0.0 \\
\hline & & 2 & 3.24819758 & 3.14159265 & 0.41901548 & 3.14159265 \\
\hline & & 3 & 4.82009921 & 3.14159265 & 0.38040647 & 0.0 \\
\hline & & 4 & 6.16330736 & 3.14159265 & 0.35790794 & 3.14159265 \\
\hline & & 5 & 7.37217726 & 3.14159265 & 0.34230124 & 0.0 \\
\hline & & 6 & 8.48848673 & 3.14159265 & 0.33047623 & 3.14159265 \\
\hline & & 7 & 9.53544905 & 3.14159265 & 0.32102229 & 0.0 \\
\hline & & 8 & 10.52766040 & 3.14159265 & 0.31318539 & 3.14159265 \\
\hline & & 9 & 11.47505663 & 3.14159265 & 0.30651729 & 0.0 \\
\hline & & 10 & 12.38478837 & 3.14159265 & 0.30073083 & 3.14159265 \\
\hline \multirow[t]{10}{*}{2} & \multirow[t]{10}{*}{1} & 1 & 1.14394586 & 2.19693240 & 0.41062389 & -0.34465562 \\
\hline & & 2 & 2.78994738 & 2.33371004 & 0.28268036 & 2.75973248 \\
\hline & & 3 & 3.75466841 & 2.34386350 & 0.24446498 & -0.38660739 \\
\hline & & 4 & 4.51583865 & 2.34768401 & 0.22308801 & 2.75312385 \\
\hline & & 5 & 5.16550405 & 2.34969423 & 0.20865176 & -0.38946020 \\
\hline & & 6 & 5.74191810 & 2.35093543 & 0.19793147 & 2.75151711 \\
\hline & & 7 & 6.26542401 & 2.35177832 & 0.18949798 & -0.39049455 \\
\hline & & 8 & 6.74838513 & 2.35238821 & 0.18260067 & 2.75079443 \\
\hline & & 9 & 7.19898515 & 2.35285002 & 0.17679969 & -0.39102840 \\
\hline & & 10 & 7.62297506 & 2.35321186 & 0.17181663 & 2.75038379 \\
\hline \multirow[t]{10}{*}{3} & \multirow[t]{10}{*}{1} & 1 & 1.21759802 & 1.69477672 & 0.29735001 & -0.54492817 \\
\hline & & 2 & 2.48297207 & 1.85987246 & 0.18961006 & 2.53138090 \\
\hline & & 3 & 3.15018496 & 1.87123995 & 0.15925753 & -0.61816012 \\
\hline & & 4 & 3.65185588 & 1.87549610 & 0.14269055 & 2.52032542 \\
\hline & & 5 & 4.06658703 & 1.87773243 & 0.13168124 & -0.62292067 \\
\hline & & 6 & 4.42582042 & 1.87911244 & 0.12360390 & 2.51764611 \\
\hline & & 7 & 4.74584133 & 1.88004930 & 0.11731083 & -0.62464498 \\
\hline & & 8 & 5.03634639 & 1.88072707 & 0.11220557 & 2.51644156 \\
\hline & & 9 & 5.30364706 & 1.88124022 & 0.10794155 & -0.62553469 \\
\hline & & 10 & 5.55211563 & 1.88164226 & 0.10430103 & 2.51575722 \\
\hline \multirow[t]{5}{*}{4} & \multirow[t]{5}{*}{1} & 1 & 1.25505627 & 1.38107258 & 0.21927675 & -0.67640886 \\
\hline & & 2 & 2.26657314 & 1.54686212 & 0.13283166 & 2.37920444 \\
\hline & & 3 & 2.76452759 & 1.55773665 & 0.10941121 & -0.77250581 \\
\hline & & 4 & 3.12701254 & 1.56179376 & 0.09683709 & 2.36514064 \\
\hline & & 5 & 3.42034992 & 1.56392339 & 0.08857070 & -0.77855062 \\
\hline
\end{tabular}


TABLE 2 (Continued)

ZEROS OF $\mathrm{AN}^{\prime}(\mathrm{Z})$

AN(Z) AT THESE ZEROS

\begin{tabular}{|c|c|c|c|c|c|c|}
\hline N & I & $\mathbf{K}$ & ABSOLUTE VALUE & ARGUMENT & ABSOLUTE VALUE & ARGUMENT \\
\hline \multirow[t]{5}{*}{4} & 1 & 6 & 3.67038911 & 1.56523700 & 0.08255454 & 2.36174030 \\
\hline & & 7 & 3.89028283 & 1.56612861 & 0.07789754 & -0.78073847 \\
\hline & & 8 & 4.08775570 & 1.56677355 & 0.07413990 & 2.36021212 \\
\hline & & 9 & 4.26777933 & 1.56726181 & 0.07101598 & -0.78186714 \\
\hline & & 10 & 4.43376503 & 1.56764432 & 0.06835968 & 2.35934401 \\
\hline \multirow[t]{10}{*}{4} & 2 & 1 & 1.16911645 & 2.55675445 & 0.31636516 & -0.23148051 \\
\hline & & 2 & 2.26471167 & 2.61171567 & 0.18799041 & 2.88582741 \\
\hline & & 3 & 2.76386315 & 2.61457241 & 0.15476664 & -0.25842185 \\
\hline & & 4 & 3.12665697 & 2.61563593 & 0.13696361 & 2.88213642 \\
\hline & & 5 & 3.42012363 & 2.61619390 & 0.12526609 & -0.26000606 \\
\hline & & 6 & 3.67023036 & 2.61653799 & 0.11675476 & 2.88124561 \\
\hline & & 7 & 3.89016427 & 2.61677152 & 0.11016710 & -0.26057913 \\
\hline & & 8 & 4.08766320 & 2.61694043 & 0.10485201 & 2.88084537 \\
\hline & & 9 & 4.26770478 & 2.61706830 & 0.10043351 & -0.26087472 \\
\hline & & 10 & 4.43370345 & 2.61716847 & 0.09667652 & 2.88061802 \\
\hline \multirow[t]{10}{*}{5} & 1 & 1 & 1.27242116 & 1.16595921 & 0.16638281 & -0.76947374 \\
\hline & & 2 & 2.10676942 & 1.32435314 & 0.09713985 & 2.27046306 \\
\hline & & 3 & 2.49818276 & 1.33438802 & 0.07890160 & -0.88278284 \\
\hline & & 4 & 2.77656148 & 1.33812180 & 0.06922535 & 2.25427255 \\
\hline & & 5 & 2.99841912 & 1.34008025 & 0.06291299 & -0.88973185 \\
\hline & & 6 & 3.18537085 & 1.34128789 & 0.05834539 & 2.25036515 \\
\hline & & 7 & 3.34827519 & 1.34210743 & 0.05482602 & -0.89224551 \\
\hline & & 8 & 3.49344679 & 1.34270019 & 0.05199725 & 2.24860955 \\
\hline & & 9 & 3.62491713 & 1.34314892 & 0.04965334 & -0.89354208 \\
\hline & & 10 & 3.74543360 & 1.34350045 & 0.04766608 & 2.24761235 \\
\hline \multirow[t]{10}{*}{5} & 2 & 1 & 1.18706890 & 2.15706794 & 0.25556171 & -0.39712288 \\
\hline & & 2 & 2.10518574 & 2.23571679 & 0.14573409 & 2.70273029 \\
\hline & & 3 & 2.49763577 & 2.23949112 & 0.11830408 & -0.44324308 \\
\hline & & 4 & 2.77627419 & 2.24089225 & 0.10378147 & 2.69664706 \\
\hline & & 5 & 2.99823868 & 2.24162675 & 0.09431314 & -0.44585010 \\
\hline & & 6 & 3.18524557 & 2.24207958 & 0.08746360 & 2.69518175 \\
\hline & & 7 & 3.34818241 & 2.24238684 & 0.08218665 & -0.44679258 \\
\hline & & 8 & 3.49337491 & 2.24260906 & 0.07794551 & 2.69452356 \\
\hline & & 9 & 3.62485957 & 2.24277728 & 0.07443148 & -0.44727864 \\
\hline & & 10 & 3.74538631 & 2.24290906 & 0.07145224 & 2.69414973 \\
\hline
\end{tabular}


TABLE 3

ZEROS OF BN(Z)

BN' $^{\prime}(Z)$ AT THESE ZEROS

\begin{tabular}{|c|c|c|c|c|c|c|}
\hline $\mathbf{N}$ & I & $\mathrm{K}$ & ABSOLUTE VALUE & ARGUMENT & ABSOLUTE VALUE & ARGUMENT \\
\hline \multirow[t]{10}{*}{1} & \multirow[t]{10}{*}{1} & 1 & 2.35387338 & 1.14253251 & 0.99310685 & 2.64060025 \\
\hline & & 2 & 4.09328731 & 1.08898311 & 1.13612833 & -0.51328287 \\
\hline & & 3 & 5.52350350 & 1.07387810 & 1.22374379 & 2.62462836 \\
\hline & & 4 & 6.78865953 & 1.06678453 & 1.28822926 & -0.51871638 \\
\hline & & 5 & 7.94555902 & 1.06266837 & 1.33979477 & 2.62185446 \\
\hline & & 6 & 9.02375637 & 1.05998103 & 1.38303390 & -0.52040694 \\
\hline & & 7 & 10.04106737 & 1.05808881 & 1.42042535 & 2.62071419 \\
\hline & & 8 & 11.00926726 & 1.05668440 & 1.45346646 & -0.52122872 \\
\hline & & 9 & 11.93664761 & 1.05560073 & 1.48313457 & 2.62009352 \\
\hline & & 10 & 12.82932394 & 1.05473921 & 1.51010464 & -0.52171419 \\
\hline \multirow[t]{10}{*}{2} & \multirow[t]{10}{*}{1} & 1 & 2.19034316 & 0.82106172 & 1.41375548 & 2.76589331 \\
\hline & & 2 & 3.32181119 & 0.80105881 & 1.73189792 & -0.38495692 \\
\hline & & 3 & 4.15977718 & 0.79540096 & 1.93641714 & 2.75387089 \\
\hline & & 4 & 4.85595843 & 0.79274231 & 2.09160568 & -0.38903670 \\
\hline & & 5 & 5.46440016 & 0.79119924 & 2.21850023 & 2.75178930 \\
\hline & & 6 & 6.01168328 & 0.79019170 & 2.32679242 & -0.39030504 \\
\hline & & 7 & 6.51319779 & 0.78948222 & 2.42180838 & 2.75093391 \\
\hline & & 8 & 6.97879729 & 0.78895562 & 2.50681386 & -0.39092147 \\
\hline & & 9 & 7.41523962 & 0.78854928 & 2.58396740 & 2.75046835 \\
\hline & & 10 & 7.82739765 & 0.78822623 & 2.65477619 & -0.39128561 \\
\hline \multirow[t]{10}{*}{3} & \multirow[t]{10}{*}{1} & 1 & 2.04746194 & 0.64572426 & 1.75035475 & 2.83991982 \\
\hline & & 2 & 2.85676075 & 0.63597601 & 2.33384735 & -0.30847649 \\
\hline & & 3 & 3.41998530 & 0.63321098 & 2.55417371 & 2.83108624 \\
\hline & & 4 & 3.87067094 & 0.63191097 & 2.80179104 & -0.31147157 \\
\hline & & 5 & 4.25401905 & 0.63115629 & 3.00701098 & 2.82955841 \\
\hline & & 6 & 4.59157685 & 0.63066347 & 3.18401245 & -0.31240243 \\
\hline & & 7 & 4.89553466 & 0.63031643 & 3.34067692 & 2.82893066 \\
\hline & & 8 & 5.17355431 & 0.63005884 & 4.48188149 & -0.31285480 \\
\hline & & 9 & 5.43080965 & 0.62986006 & 3.61087469 & 2.82858900 \\
\hline & & 10 & 5.67098195 & 0.62970203 & 3.72993990 & -0.31312202 \\
\hline \multirow[t]{5}{*}{4} & \multirow[t]{5}{*}{1} & 1 & 1.93112085 & 0.53342839 & 2.0440945 & 2.88921621 \\
\hline & & 2 & 2.54860724 & 0.52792834 & 2.68089039 & -0.25751312 \\
\hline & & 3 & 2.96087343 & 0.52636551 & 3.11140074 & 2.88254816 \\
\hline & & 4 & 3.28260643 & 0.52563046 & 3.44834572 & -0.25977247 \\
\hline & & 5 & 3.55136466 & 0.52520370 & 3.73012266 & 2.88139582 \\
\hline
\end{tabular}


TABLE 3 (Continued)

ZEROS OF BN(Z)

BN $^{\prime}(Z)$ AT THESE ZEROS

\begin{tabular}{|c|c|c|c|c|c|c|}
\hline $\mathbf{N}$ & I & K & ABSOLUTE VALUE & ARGUMENT & ABSOLUTE VALUE & ARGUMENT \\
\hline \multirow[t]{5}{*}{4} & 1 & 6 & 3.78468721 & 0.52492500 & 3.97487884 & -0.26047450 \\
\hline & & 7 & 3.99234607 & 0.52472873 & 4.19277919 & 2.88092239 \\
\hline & & 8 & 4.18040807 & 0.52458305 & 4. 39015272 & -0.26081566 \\
\hline & & 9 & 4.35292805 & 0.52447063 & 4.57123677 & 2.88066474 \\
\hline & & 10 & 4.51276562 & 0.52438126 & 4.73902302 & -0.26101718 \\
\hline \multirow[t]{10}{*}{4} & 2 & 1 & 1.93480107 & 1.60819828 & 1.44785568 & 2.39206826 \\
\hline & & 2 & 2.54957321 & 1.58731788 & 1.89637381 & -0.76904130 \\
\hline & & 3 & 2.96133373 & 1.58135876 & 2.20043031 & 2.36671181 \\
\hline & & 4 & 3.28288202 & 1.57855369 & 2.43855193 & -0.77765897 \\
\hline & & 5 & $3.5515508 ?$ & 1.57692461 & 2.63773270 & 2.36231376 \\
\hline & & 6 & 3.78482274 & 1.57586059 & 2.81076413 & -0.78033900 \\
\hline & & 7 & 3.99244987 & 1.57511122 & 2.96481952 & 2.36050622 \\
\hline & & 8 & 4.18049055 & 1.57455496 & 3.10436791 & -0.78164162 \\
\hline & & 9 & 4.35299545 & 1.57412570 & 3.23240250 & 2.35952241 \\
\hline & & 10 & 4.51282190 & 1.57378442 & 3.35103707 & -0.78241113 \\
\hline \multirow[t]{10}{*}{5} & 1 & 1 & 1.83719462 & 0.45490143 & 2.30987514 & 2.92451728 \\
\hline & & 2 & 2.33022083 & 0.45148891 & 3.08913616 & -0.22106945 \\
\hline & & 3 & 2.64975572 & 0.45051812 & 3.62362660 & 2.91933328 \\
\hline & & 4 & 2.89469697 & 0.45006143 & 4.04567630 & -0.22282494 \\
\hline & & 5 & 3.09668044 & 0.44979626 & 4.40089972 & 2.91843805 \\
\hline & & 6 & 3.27026439 & 0.44962309 & 4.71101512 & -0.22337031 \\
\hline & & 7 & 3.42347193 & 0.44950113 & 4.98825473 & 2.91807028 \\
\hline & & 8 & 3.56124063 & 0.44941060 & 5.24026829 & -0.22363533 \\
\hline & & 9 & 3.68684643 & 0.44934074 & 5.47219632 & 2.91787013 \\
\hline & & 10 & 3.80258501 & 0.44928520 & 5.68767972 & -0.22379187 \\
\hline \multirow[t]{10}{*}{5} & 2 & 1 & 1.83852512 & 1.36790487 & 1.82539101 & 2.49421373 \\
\hline & & 2 & 2.33055656 & 1.35588960 & 2.43965146 & -0.66144673 \\
\hline & & 3 & 2.64991232 & 1.35246493 & $2: 86147404$ & 2.47594806 \\
\hline & & 4 & 2.89478936 & 1.35085326 & 3.19464804 & -0.66764051 \\
\hline & & 5 & 3.09674215 & 1.34991733 & 3.47509693 & 2.47278858 \\
\hline & & 6 & 3.27030891 & 1.34930607 & 3.71994522 & -0.66956545 \\
\hline & & 7 & 3.42350577 & 1.34887557 & 3.93884286 & 2.47149043 \\
\hline & & 8 & 3.56126734 & 1.34855601 & 4.13782639 & -0.67050093 \\
\hline & & 9 & 3.68686813 & 1.34830942 & 4.32095296 & 2.47078393 \\
\hline & & 10 & 3.80260304 & 1.34811336 & 4.49109644 & -0.67105351 \\
\hline
\end{tabular}


TABLE 4

ZEROS OF BN' (Z)

BN(Z) AT THESE ZEROS

\begin{tabular}{|c|c|c|c|c|c|c|}
\hline $\mathrm{N}$ & I & $\mathbf{K}$ & ABSOLUTE VALUE & ARGUMENT & ABSOLUTE VALUE & ARGUMENT \\
\hline \multirow[t]{10}{*}{1} & \multirow[t]{10}{*}{1} & 1 & 1.12139329 & 1.37792417 & 0.75004149 & 0.46597789 \\
\hline & & 2 & 3.25690823 & 1.10658749 & 0.59221663 & -2.63235403 \\
\hline & & 3 & 4.82400261 & 1.07998319 & 0.53787063 & 0.51549330 \\
\hline & & 4 & 6.16568666 & 1.06986001 & 0.50611020 & -2.62362859 \\
\hline & & 5 & 7.37383799 & 1.06451720 & 0.48406006 & 0.51928282 \\
\hline & & 6 & 8.48973856 & 1.06121408 & 0.46734684 & -2.62149057 \\
\hline & & 7 & 9.53644071 & 1.05896948 & 0.45398232 & 0.52066021 \\
\hline & & 8 & 10.52847375 & 1.05734473 & 0.44290250 & -2.62052784 \\
\hline & & 9 & 11.47574112 & 1.05611417 & 0.43347447 & 0.52137155 \\
\hline & & 10 & 12.38537593 & 1.05514984 & 0.42529258 & -2.61998058 \\
\hline \multirow[t]{10}{*}{2} & \multirow[t]{10}{*}{1} & 1 & 1.14394586 & 0.94466026 & 0.82124778 & 0.34465562 \\
\hline & & 2 & 2.78994738 & 0.80788261 & 0.56536073 & -2.75973248 \\
\hline & & 3 & 3.75466841 & 0.79772916 & 0.48892996 & 0.38660739 \\
\hline & & 4 & 4.51583865 & 0.79390865 & 0.44617601 & -2.75312385 \\
\hline & & 5 & 5.16550405 & 0.79189845 & 0.41730352 & 0.38946020 \\
\hline & & 6 & 5.74191810 & 0.79065722 & 0.39586294 & -2.75151711 \\
\hline & & 7 & 6.26542401 & 0.78981433 & 0.37899595 & 0.39049455 \\
\hline & & 8 & 6.74838513 & 0.78920444 & 0.36520134 & -2.75079443 \\
\hline & & 9 & 7.19898515 & 0.78874263 & 0.35359938 & 0.39102840 \\
\hline & & 10 & 7.62297506 & 0.78838079 & 0.34363326 & -2.75038379 \\
\hline \multirow[t]{10}{*}{3} & \multirow[t]{10}{*}{1} & 1 & 1.16184363 & 0.72171921 & 0.80837790 & 0.27622099 \\
\hline & & 2 & 2.48136268 & 0.63937810 & 0.51027252 & -2.83541560 \\
\hline & & 3 & 3.14958342 & 0.63436113 & 0.42846095 & 0.30968393 \\
\hline & & 4 & 3.65152544 & 0.63248531 & 0.38386230 & -2.83053938 \\
\hline & & 5 & 4.06637280 & 0.63150004 & 0.35423559 & 0.31178173 \\
\hline & & 6 & 4.42566796 & 0.63089212 & 0.33250229 & -2.82935901 \\
\hline & & 7 & 4.74572612 & 0.63047945 & 0.31557117 & 0.31254129 \\
\hline & & 8 & 5.03625559 & 0.63018091 & 0.30183638 & -2.82882843 \\
\hline & & 9 & 5.30357325 & 0.62995489 & 0.29036516 & 0.31293318 \\
\hline & & 10 & 5.55205419 & 0.62977782 & 0.28057148 & -2.82852701 \\
\hline \multirow[t]{5}{*}{4} & \multirow[t]{5}{*}{1} & 1 & 1.16911645 & 0.58483820 & 0.77493322 & 0.23148051 \\
\hline & & 2 & 2.26471167 & 0.52987698 & 0.46048057 & -2.88582741 \\
\hline & & 3 & 2.76386315 & 0.52702025 & 0.37909929 & 0.25842185 \\
\hline & & 4 & 3.12665697 & 0.52595672 & 0.33549096 & -2.88213642 \\
\hline & & 5 & 3.42012363 & 0.52539876 & 0.30683799 & 0.26000606 \\
\hline
\end{tabular}


TABLE 4 (Continued)

ZEROS OF BN'(Z)

\begin{tabular}{|c|c|c|c|c|c|c|}
\hline $\mathbf{N}$ & I & K & ABSOLUTE VALUE & ARGUMENT & ABSOLUTE VALUE & ARGUMENT \\
\hline \multirow[t]{5}{*}{4} & 1 & 6 & 3.67023036 & 0.52505466 & 0.28598958 & -2.88124561 \\
\hline & & 7 & 3.89016427 & 0.52482114 & 0.26985318 & 0.26057913 \\
\hline & & 8 & 4.08766320 & 0.52465223 & 0.25683393 & -2.88084537 \\
\hline & & 9 & 4.26770478 & 0.52452436 & 0.24601085 & 0.26087472 \\
\hline & & 10 & 4.43370345 & 0.52442418 & 0.23680815 & -2.88061802 \\
\hline \multirow[t]{10}{*}{4} & 2 & 1 & 1.25505627 & 1.76052008 & 0.53711614 & 0.67640886 \\
\hline & & 2 & 2.26657314 & 1.59473053 & 0.32536980 & -2.37920444 \\
\hline & & 3 & 2.76452759 & 1.58385600 & 0.26800164 & 0.77250581 \\
\hline & & 4 & 3.12701254 & 1.57979890 & 0.23720146 & -2.36514064 \\
\hline & & 5 & 3.42034992 & 1.57766926 & 0.21695303 & 0.77855062 \\
\hline & & 6 & 3.67038911 & 1.57635565 & 0.20221649 & -2.36174030 \\
\hline & & 7 & 3.89028283 & 1.57546405 & 0.19080923 & 0.78073847 \\
\hline & & 8 & 4.08775570 & 1.57481910 & 0.18160492 & -2.36021212 \\
\hline & & 9 & 4.26777933 & 1.57433084 & 0.17395291 & 0.78186714 \\
\hline & & 10 & 4.43376503 & 1.57394833 & 0.16744633 & -2.35934401 \\
\hline \multirow[t]{10}{*}{5} & 1 & 1 & 1.17023190 & 0.49197486 & 0.73788630 & 0.19968410 \\
\hline & & 2 & 2.10498408 & 0.45271092 & 0.41924569 & -2.92188880 \\
\hline & & 3 & 2.49756627 & 0.45092690 & 0.34031050 & 0.22177436 \\
\hline & & 4 & 2.77623770 & 0.45026482 & 0.29852995 & -2.91901381 \\
\hline & & 5 & 2.99821577 & 0.44991777 & 0.27129226 & 0.22300622 \\
\hline & & 6 & 3.18522966 & 0.44970382 & 0.25158868 & -2.91832146 \\
\hline & & 7 & 3.34817063 & 0.44955864 & 0.23640913 & 0.22345153 \\
\hline & & 8 & 3.49336577 & 0.44945365 & 0.22420928 & -2.91801048 \\
\hline & & 9 & 3.62485226 & 0.44937417 & 0.21410106 & 0.22368118 \\
\hline & & 10 & 3.74538031 & 0.4493119 & 0.20553121 & -2.91783386 \\
\hline \multirow[t]{10}{*}{5} & 2 & 1 & 1.21849446 & 1.47849557 & 0.57588393 & 0.58912099 \\
\hline & & 2 & 2.10564782 & 1.36019593 & 0.33092584 & -2.48495960 \\
\hline & & 3 & 2.49779516 & 1.35390716 & 0.26868422 & 0.66393331 \\
\hline & & 4 & 2.77635789 & 1.35157105 & 0.23571083 & -2.47482066 \\
\hline & & 5 & 2.99829124 & 1.35034619 & 0.21420942 & 0.66828033 \\
\hline & & 6 & 3.18528207 & 1.34959102 & 0.19865382 & -2.47237706 \\
\hline & & 7 & 3.34820944 & 1.34907859 & 0.18666920 & 0.66985213 \\
\hline & & 8 & 3.49339585 & 1.34870796 & 0.17703682 & -2.47127935 \\
\hline & & 9 & 3.62487634 & 1.34842741 & 0.16905573 & 0.67066279 \\
\hline & & 10 & 3.74540009 & 1.34820763 & 0.16228919 & -2.47065588 \\
\hline
\end{tabular}

